\title{
Oxidative Stress is Independent Factor for End-stage Renal Disease in Type 2 Diabetes Mellitus Patients
}

\author{
Kirti Balyan*, Pragya Sharma*, Kailash Chandra, Sarita Agarwal, S.K. Jain \\ Department of Biochemistry, Hamdard Institute of Medical Sciences and Research, \\ Jamia Hamdard, New Delhi.
}

\begin{abstract}
An imbalance between oxidant and antioxidants is thought to precede the development of renal lesions. The aim of present study is to determine the relationship between oxidative markers and severity of microalbuminuria in patients with type 2 diabetes mellitus (T2DM). A total of 100 T2DM patients (50 males and 50 females) participated in this study. They were screened for microalbuminuria along with oxidative status in patients. Microalbuminuria was detected by measuring the albumin to creatinine ratio (ACR) in urine samples. Patients were divided into two groups; normoalbuminuria $(n=36)$ and microalbuminuria $(n=64)$ as per the ACR levels. No difference in the groups was observed in terms of age, sex, glycated hemoglobin (HbAlc) and blood pressure. The level of oxidative stress was significantly higher in microalbuminuria group of T2DM patients. A significant correlation was observed between ACR and lipid peroxidation (MDA) levels. We conclude that oxidative stress is one of the important mediators of end-stage renal disease (ESRD).
\end{abstract}

Keywords: Type 2 diabetes mellitus, microalbuminuria, diabetic nephropathy, albumin to creatinine ratio, oxidative stress.

\section{Introduction}

The incidence of diabetes mellitus is increasing globally. Besides, it is also one of the leading causes of end-stage renal disease (ESRD). According to the International Diabetes Federation (IDF), approximately 425 million adults are presently living with diabetes and by 2045, this will reach to 629 million (1). Type 2 diabetes mellitus (T2DM) is associated with older age, obesity, family history of diabetes, impaired glucose metabolism, and physical inactivity. The disease usually begins with either insufficient insulin secretion or its impaired action. Uncontrolled diabetes leads to a number of short and long-term complications such as ketoacidosis, diabetic retinopathy, nephropathy, neuropathy, and many other complications.
Renal damage (nephropathy) is a serious complication of diabetes mellitus and is the leading cause of ESRD. In diabetes, kidney disease is characterized by progressive albuminuria, decline in glomerular filtration rate (GFR), hypertension and high risk of cardiovascular mortality and morbidity (2). Microalbuminuria is an early marker of progressive nephropathy that can be measured in spot urine as albumin to creatinine ratio (ACR). In general, ACR has slightly better diagnostic accuracy than urine albumin concentration alone for detection of albuminuria in many populations (3). Moreover, ACR appears to be the best marker for early identification of diabetic nephropathy. Screening for albuminuria is recommended in patients at increased risk for chronic kidney disease (CKD), those with

Correspondence: Dr. S.K. Jain, Professor, Department of Biochemistry, Hamdard Institute of Medical Sciences and Research, Jamia Hamdard, New Delhi-110062. Mob: +919818608273. Fax: 26059663. Email: skjain@jamiahamdard.ac.in.*equal contribution. 
hypertension, diabetes mellitus, cardiovascular disease and a family history of CKD.

Diabetic nephropathy is characterized by excessive accumulation of extracellular matrix in kidney. The basement membranes are thickened and the glomerular mesangial matrix and the tubulointerstitial space are expanded. Increased oxidative stress in diabetes contributes to the pathogenesis of diabetic nephropathy and its progression to ESRD. However, relatively little information is available to define the association between oxidative stress and microalbuminuria in human subjects. It is, therefore, pertinent to understand the inter-relationship between oxidative markers and microalbuminuria in $\mathrm{T} 2 \mathrm{DM}$ patients. It would be clinically useful to identify the associations of oxidative stress with severity of microalbuminuria, so that it can be used as a determining factor for assessing nephropathy in patients with diabetes.

\section{Materials and Methods}

A total of 100 T2DM patients (50 males and 50 females) were recruited from the medical OPD of HAHC Hospital from July 2017 to May 2018. T2DM was diagnosed as per the criteria laid down by the American Diabetes Association (ADA) (4). Based on their clinical history, subjects with the following manifestations were excluded from the study: patients with high blood pressure, other causes of albuminuria such as fever, urinary tract infection, congestive heart disease, and hematuria. The anthropometric data were collected for each participant at the time of recruitment and informed consent was obtained. The study was ethically approved by the Institutional Ethics Committee (IEC) of Jamia Hamdard, New Delhi.

\section{Study Assessment}

After an overnight fasting, blood and urine samples were collected for biochemical analysis. The biochemical parameters such as fasting glucose level, lipid profile, liver, and kidney function tests were determined using Siemens Xpand plus biochemistry autoanalyzer. The glycated hemoglobin (HbAlc) level was evaluated in Bio-Rad D-10 (TM ${ }^{(\mathrm{T}}$ high-performance liquid chromatography-based system. The oxidative markers such as reduced glutathione (GSH), catalase, superoxide dismutase (SOD) and malondialdehyde were analyzed by spectrophotometer using standard protocols(5).

\section{Albumin-creatinine Ratio (ACR) Measurement}

Urinary albumin and creatinine levels were analyzed in random spot urine samples by Siemens Xpand plus biochemistry autoanalyzer and ACR was calculated. ACR is considered as an early marker of progressive nephropathy. As per ADA and National Kidney Foundation guidelines, microalbuminuria is defined by urinary ACR level at the range in between 30 to $300 \mu \mathrm{g} / \mathrm{mg}$ in both men and women(3).

\section{Statistical Analysis}

For each variable, values were expressed as mean $\pm \mathrm{SEM}$. The statistical analysis was carried out in GraphPad Prism, ver. 5.0 software. To detect possible relationships between ACR and other variables, Pearson's correlation coefficient (r) was calculated. At 95\% confidence interval, $\mathrm{p}<0.05$ was considered as statistically significant.

\section{Results}

In present study, out of $100 \mathrm{~T} 2 \mathrm{DM}$ patients, $64 \%$ of patients were diagnosed to have microalbuminuria and rest $36 \%$ had normal albumin levels (normoalbuminuria) (Table 1). Moreover, $36.1 \%$ and $42.1 \%$ of smokers were present in the normoalbuminuria and microalbuminuria groups, respectively. No differences in the groups were observed in terms of age, sex, HbAlc level and blood pressure.

The biochemical parameters such as total cholesterol, triglyceride, high-density 
Table 1: Baseline characteristics of patients with type 2 diabetes mellitus

\begin{tabular}{|l|c|c|}
\hline & $\begin{array}{c}\text { Normoalbuminuria } \\
(\mathbf{n = 3 6 )}\end{array}$ & $\begin{array}{c}\text { Microalbuminuria } \\
(\mathbf{n}=\mathbf{6 4})\end{array}$ \\
\hline Sex $(\mathrm{M} / \mathrm{F})$ & $14 / 22$ & $33 / 31$ \\
\hline Smoker/non-smoker $(\mathrm{n})$ & $13 / 23$ & $27 / 37$ \\
\hline Alcoholic/non-alcoholic $(\mathrm{n})$ & $7 / 29$ & $13 / 51$ \\
\hline Age $($ years $)$ & $48.5 \pm 13.3$ & $46.1 \pm 14.3$ \\
\hline SBP/DBP $(\mathrm{mmHg})$ & $128.4 \pm 8.1 / 83.3 \pm 5.1$ & $131.4 \pm 7.4 / 83.2 \pm 5.5$ \\
\hline HbAlc $(\%)$ & $10.4 \pm 1.5$ & $10.8 \pm 1.93$ \\
\hline ACR $(\mu \mathrm{g} / \mathrm{mg})$ & $14.06 \pm 5.2$ & $120.8 \pm 80$ \\
\hline
\end{tabular}

SBP-systolic blood pressure; DBP- Diastolic blood pressure; ACR-Albumin-creatinine ratio. All values are expressed as mean $\pm \mathrm{SD} ; \mathrm{p}<0.05$ statistical significant.

Table 2: Biochemical parameters in T2DM patients in normoalbuminuria and microalbuminuria groups

\begin{tabular}{|l|c|c|}
\hline & $\begin{array}{c}\text { Normoalbuminuria } \\
(\mathbf{n}=\mathbf{3 6})\end{array}$ & $\begin{array}{c}\text { Microalbuminuria } \\
(\mathbf{n}=\mathbf{6 4})\end{array}$ \\
\hline Total cholesterol $(\mathrm{mg} / \mathrm{dL})$ & $218.9 \pm 30.2$ & $219.3 \pm 30.7^{\mathrm{ns}}$ \\
\hline Triglyceride $(\mathrm{mg} / \mathrm{dL})$ & $194.6 \pm 25.1$ & $186.5 \pm 26.7^{\mathrm{ns}}$ \\
\hline HDL-C $(\mathrm{mg} / \mathrm{dL})$ & $35.3 \pm 6.4$ & $33.8 \pm 4.4^{\mathrm{ns}}$ \\
\hline LDL-C $(\mathrm{mg} / \mathrm{dL})$ & $149.0 \pm 19.3$ & $143.2 \pm 21.4^{\mathrm{ns}}$ \\
\hline VLDL-C $(\mathrm{mg} / \mathrm{dL})$ & $38.9 \pm 5.8$ & $37.3 \pm 8.6^{\mathrm{ns}}$ \\
\hline SOD $(\mathrm{U} / \mathrm{mL})$ & $6.58 \pm 3.6$ & $5.20 \pm 3.8^{*}$ \\
\hline GSH $(\mu \mathrm{mol})$ & $62.3 \pm 10.1$ & $54.6 \pm 9.2^{\mathrm{ns}}$ \\
\hline MDA $(\mu \mathrm{mol})$ & $174.3 \pm 30.1$ & $216.0 \pm 32.2^{* *}$ \\
\hline Catalase $(\mathrm{KU})$ & $178.1 \pm 28.2$ & $175.1 \pm 25.2^{\mathrm{ns}}$ \\
\hline
\end{tabular}

Data were expressed as mean \pm SEM. HDL-C- High density lipoprotein-cholesterol; LDL- Low density lipoprotein, VLDL- Very low density lipoprotein, SOD- Superoxide dismutase, GSH- Glutathione; MDA-Malondialdehyde. $\mathrm{p}>0.05$ [not significant (ns)], ${ }^{*} \mathrm{p}<0.05, * * \mathrm{p}<0.001$. 
lipoprotein cholesterol (HDL-C), and lowdensity lipoprotein-cholesterol (LDL-C) were analyzed in T 2 DM patients of normoalbuminuria and microalbuminuria groups. No statistically significant difference was observed in these parameters between the two groups (Table 2). The assessment of oxidative stress revealed a significantly lower levels of antioxidant enzymes in patients with microalbuminuria than the normoalbuminuria. Lipid peroxidation values were significantly higher in diabetic patients with microalbuminuria as compared to normoalbuminuria. Reduced GSH level in plasma did not show any statistically significant difference amongst the groups, however the values in microalbuminuria group were slightly lower as compared to normoalbuminuria group.

Further, to decipher the relation of different associated variables with microalbuminuria, a correlation study was carried out by using

Table 3: A correlation study in between albumin-creatinine ratio (ACR) and other associated factors

\begin{tabular}{|c|c|c|}
\hline & $\begin{array}{c}\text { Correlation coefficient } \\
(\mathbf{r})\end{array}$ & p value \\
\hline Age (years) & 0.12 & 0.34 \\
\hline Sex & 0.11 & 0.36 \\
\hline $\mathrm{SOD}(\mathrm{U} / \mathrm{mL})$ & -0.10 & 0.43 \\
\hline $\mathrm{GSH}(\mu \mathrm{mol})$ & -0.11 & 0.36 \\
\hline Catalase $(\mathrm{KU})$ & 0.001 & 0.99 \\
\hline
\end{tabular}

SBP-systolic blood pressure; DBP- Diastolic blood pressure;

ACR-Albumin-creatinine ratio. All values are expressed as mean $\pm S D ; p<0.05$ statistical significant.

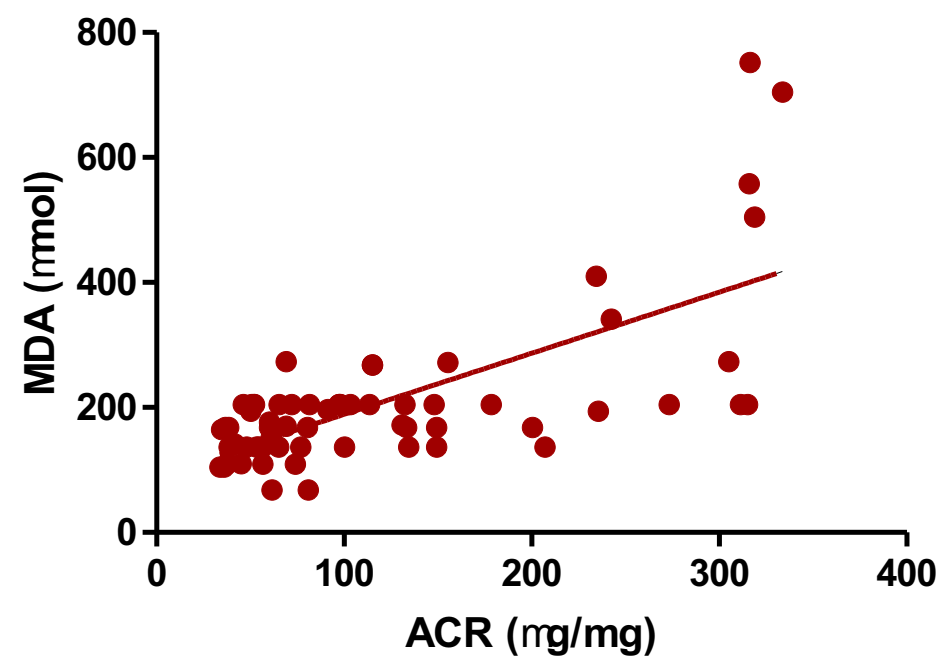

Fig. 1: A correlation study (Pearson) in between albumin-creatinine ratio and plasma

MDA levels in T2DM patients. $(r+0.69, p<0.001)$.

ACR-Albumin-creatinine ratio; MDA- Malondialdehyde.

+ value showed positive correlation, $\mathrm{p}$ value $<0.05$ is significant correlation. 
GraphPad Prism, ver. 5.0 software. In this study, only malondialdehdyde levels showed a positive correlation with ACR $(\mathrm{r}+0.69, \mathrm{p}<0.001)$ (Table 3 and Fig. 1).

\section{Discussion}

The outcome of this study suggests that oxidative stress is one of the mediators related to vascular complications like diabetic nephropathy in T2DM patients. We excluded the hypertensive patients in this study as it is a wellestablished confounding factor for ESRD in T2DM patient (6). However, $64 \%$ of T2DM patients were diagnosed as having microalbuminuria in this study. Microalbuminuria is a risk factor for development of ESRD in diabetic patients. ACR is an established marker for revealing microalbuminuria in patients and it was statistically increased in microalbuminuria group. The HbAlc level in microalbuminuria and normoalbuminuria group was not statistically different that indicates that glycoxidation itself does not lead to the change in the level of oxidative stress in diabetic patients. Further, total cholesterol, triglycerides, HDL-C, LDL-C and VLDL-C values also were not significantly different in microalbuminuria group of patients as compared to normoalbuminuria group $(p>0.05)$. The evaluation of oxidative stress level in both the groups of T2DM patients revealed that malondialdehyde (MDA) levels were significantly higher in microalbuminuria group as compared to normoalbuminuria group ( $p$ $<0.001)$. MDA is routinely measured as an indicator of oxidative stress. Hyperglycemia in T2DM patients resulted in the generation of ROS, increasing oxidative stress in affected tissues, which are damaged by the consequent activation of nuclear factor kappa $\mathrm{B}$, along with advanced glycation end product (AGE) formation and activation of the protein kinase $\mathrm{C}$ and hexosamine pathways that lead to the increased level of MDA in patients (6-8). The reduced GSH levels were decreased in subjects with microalbuminuria as compared to normoalbuminuria patients. A significant decreased in levels of SOD was observed in subjects of microalbuminuria group as compared to normoalbuminuria $(p<0.05)$. SOD is required for scavenging the superoxide radicals (9), therefore reduced activity of SOD leads to increased levels of superoxide radicals which will result in decreased levels of reduced GSH(10). Oxidative stress continued in diabetes by hyperglycemia and the glycoxidation products such as HbAlc and AGE and the absence of an appropriate compensatory response from the endogenous antioxidant network has been implicated in systemic endothelial dysfunction $(6,11)$. Microalbuminuria is considered a marker of endothelial dysfunction (12). The correlation study between ACR and MDA levels showed a positive correlation which proves that oxidative stress is one of the risk factors for diabetic nephropathy. The oxidative stress in diabetic patients and altered antioxidant status, cellular milieu of renal system is disrupted causing renal lesions and damages that lead to the development of diabetic nephropathy due to disruption of endothelial glycocalyx through the reactive oxygen species (ROS), cytokines and vascular endothelial growth factors $(13,14)$.

\section{Conclusion}

We conclude that diabetic patients have considerable higher risk of developing renal impairment and should be regularly monitored to expedite early detection of diabetes-induced nephropathy. Strategy for targeting the oxidative stress in T2DM patients may be an appropriate therapy for diabetic nephropathy. The outcome of this study would help in defining new targets for the prevention and treatment of diabetic nephropathy.

\section{Funding}

This work was supported by intramural funding from Hamdard Institute of Medical Sciences and Research, Jamia Hamdard, New Delhi. 


\section{Acknowledgment}

The author wish to thank Prof. Sunil Kohli, Department of Medicine, HIMSR for clinical evaluation of the subjects. The consent of the patients for inclusion in the study is gratefully acknowledged.

\section{Conflict of Interest}

We confirmed that there are no known conflicts of interest associated with this publication.

\section{References}

1. IDF Diabetes Atlas (2017), 8th edn. International Diabetes Fedration, 1-150.

2. Van Buren PN, Toto R (2011). Hypertension in diabetic nephropathy: epidemiology, mechanisms, and management. $A d v$ Chronic Kidney Dis 18(1): 28-41.

3. Bakker AJ (1999). Detection of microalbuminuria. Receiver operating characteristic curve analysis favors albumin-to-creatinine ratio over albumin concentration. Diabetes Care 22: 307-313.

4. American Diabetes Association (2010). Diagnosis and classification of diabetes mellitus. Diabetes Care 33(Suppl 1): S62-S69.

5. Chandra K, Khan W, Jetley S, Ahmad S, Jain S (2018). Antidiabetic, toxicological, and metabolomic profiling of aqueous extract of Cichorium intybus seeds. Pharmacogn Mag 14: 377.

6. Palatini P (2003). Microalbuminuria in hypertension. Curr Hypertens Rep 5: 208-214.

7. Fontana L, Eagon JC, Trujillo ME, Scherer PE, Klein S (2007). Visceral fat adipokine secretion is associated with systemic inflammation in obese humans. Diabetes 56:1010-1013.
8. Ogawa S, Nako K, Okamura M, Senda M, Mori T, Ito S (2011). Aliskiren reduces albuminuria and oxidative stress, and elevates glomerular filtration rates in Japanese patients with advanced diabetic nephropathy. Hypertens Res 34: 400-401.

9. Toblli JE, Cao G, Giani JF, Muñoz MC, Angerosa M, Dominici FP (2011). Longterm treatment with nebivolol attenuates renal damage in Zucker diabetic fatty rats. $J$ Hypertens 29: 1613-1623.

10. Wu LL, Chiou CC, Chang PY, Wu JT (2004). Urinary 8-OHdG: a marker of oxidative stress to DNA and a risk factor for cancer, atherosclerosis and diabetics. Clin Chim Acta 339: 1-9.

11. Fukami K, Ueda S, Yamagishi $\mathrm{S}$, et al (2004). AGEs activate mesangial TGF$\beta$-Smad signaling via an angiotensin II type I receptor interaction. Kidney Int 66: 2137-2147.

12. Naidoo DP (2002). The link between microalbuminuria, endothelial dysfunction and cardiovascular disease in diabetes. Cardiovasc J SAfr 13: 194-199.

13. Satchell SC, Tooke JE (2008). What is the mechanism of microalbuminuria in diabetes: a role for the glomerular endothelium? Diabetologia 51: 714-725.

14. Giugliano D, Ceriello A, Paolisso G (1996). Oxidative stress and diabetic vascular complications. Diabetes Care 19: 257-267. 